\title{
DOA Estimation Exploiting Coprime Frequencies
}

\author{
Si Qin, Yimin D. Zhang, and Moeness G. Amin \\ Center for Advanced Communications, Villanova University, Villanova, PA 19085, USA
}

\begin{abstract}
Coprime array, which utilizes a coprime pair of uniform linear subarrays, is an attractive structure to achieve sparse array configurations. Alternatively, effective coprime array configurations can be implemented using a uniform linear array with two coprime sensing frequencies. This enables the integration of the coprime array and filter concepts to achieve high capabilities in meeting system performance and complexity constraints. This paper examines its performance for direction-of-arrival estimations. In particular, we analyze the number of detectable signals and the estimation accuracy as related to the array configurations and sensing frequencies.
\end{abstract}

Keywords: Coprime array, sparse array, difference co-array, direction-of-arrival estimation

\section{INTRODUCTION}

An important application of array signal processing is direction-of-arrival (DOA) estimation, which determines the spatial spectra of the impinging electromagnetic waves. It is well known that an $N$-element uniform linear array (ULA) has $N-1$ degrees-of-freedom (DOFs), i.e., it resolves up to $N-1$ sources by using conventional DOA estimation methods, such as MUSIC and ESPRIT. ${ }^{1,2}$ On the other hand, a higher number of DOFs can be achieved to resolve more sources by using the same number of array sensors if they are sparely placed. ${ }^{3}$ Such higher DOFs are usually achieved by exploiting the extended difference co-array whose virtual sensor positions are determined by the lag differences between physical sensors. The minimum redundancy array (MRA) ${ }^{4}$ is a well-known configuration of sparse linear array which, for a given number of physical sensors, maximizes the number of consecutive virtual sensors in the resulting difference co-array. A significant problem with the MRA is that, given an arbitrary number of physical sensors, there is no systematical method to design an MRA configuration and to assess its achievable DOFs.

Recently, several methods have been proposed for systematical construction of sparse arrays. The nested array ${ }^{5}$ which is designed with two uniform linear subarrays, in which one subarray has a unit interelement spacing, can resolve $O\left(N^{2}\right)$ sources with $N$ sensors. The coprime array concept was proposed in ${ }^{6}$ to resolve $O(M N)$ sources with $M+N-1$ physical sensors. To obtained a longer consecutive set of virtual sensors, a modified coprime array structure was proposed $i^{7}$ by doubling the number of elements in the smaller subarray. Such approach is generalized in ${ }^{8}$ by introducing an integer factor that compresses the interelement spacing of one constituting subarray, and the exact number of DOFs is obtained. On the other hand, it is shown that, by exploiting sparse signal reconstruction techniques, a higher number of DOFs is achieved by utilizing the nonconsecutive virtual sensors that are otherwise unused in conventional DOA estimation approaches. ${ }^{9}$ In addition, by placing the two subarrays collinearly instead of collocated, the number of unique virtual sensors is further increased, thus providing more DOFs of the coprime array when the sparse signal reconstruction techniques are exploited. ${ }^{10}$

While the coprime array concept is developed by using two physical uniform linear subarrays, it is shown in ${ }^{9}$ that effective coprime array configurations can also be implemented using a single sparse ULA with two coprime frequencies. This can be achieved, e.g., by exploiting two continuous-wave (CW) sinusoids whose frequencies satisfy a certain coprime relationship. In this case, the ULA acts as two virtual subarrays at these two frequencies with different interelement spacing in terms of the respective wavelengths, resulting in an equivalent structure to coprime arrays. As such, it integrates the concept of coprime array and coprime filter to achieve high capabilities in meeting system performance and complexity constraints. Unlike coprime arrays wherein the numbers of subarray sensors and the interelement spacings have to satisfy the coprime relationship, only the two frequencies

Contact information: yimin.zhang@villanova.edu. 
are required to be coprime. As a result, it provides greater flexibility in system design and the capability of configuration adaptivity to the application environments. While the effectiveness of this equivalent coprime array structure is verified through simulation examples, the number of the achievable DOFs remains unclear for different choices of coprime frequency pairs.

The primary objective of this paper is to derived the analytical expression of the number of DOFs and the resolvable sources with respect to the number of physical sensors and the selected coprime frequencies. In addition, we find that the available DOFs are solely determined by the number of cross-lags between the two effective subarrays. As such, the DOA estimation problem is cast as a sparse signal reconstruction problem by using the difference co-array obtained from such cross-lags. On the other hand, the self-lags obtained for each subarray can also be exploited for improving the performance. In the latter case, because the reflection characteristics at the two frequencies generally differ due to the differences in the propagation phase and possibly the target reflectivity, group sparse signal reconstruction methods have to be used to exploit both self- and crosslags.

Notations: We use lower-case (upper-case) bold characters to denote vectors (matrices). In particular, $\mathbf{I}_{N}$ denotes the $N \times N$ identity matrix. (.)* implies complex conjugation, whereas $(.)^{T}$ and $(.)^{H}$ respectively denote the transpose and conjugate transpose of a matrix or vector. $\operatorname{vec}(\cdot)$ denotes the vectorization operator that turns a matrix into a vector by stacking all columns on top of the another, and $\operatorname{diag}(\mathbf{x})$ denotes a diagonal matrix that uses the elements of $\mathbf{x}$ as its diagonal elements. $\|\cdot\|_{2}$ and $\|\cdot\|_{1}$ respectively denote the Euclidean $\left(l_{2}\right)$ and $l_{1}$ norms, and $\mathrm{E}(\cdot)$ is the statistical expectation operator. $\bigotimes$ denotes the Kronecker product, and $\lfloor\cdot\rfloor$ denotes the largest integer not exceeding the argument.

\section{SYSTEM MODEL}

We consider a DOA estimation problem by exploiting signals at two coprime frequencies $f_{1}$ and $f_{2}$. This can be achieved either actively by simultaneously emitting two CW signals at these frequencies from a single transmit antenna or a phased array, or passively by properly filtering signals at such frequencies. The signals received from $Q$ unknown far-field targets are received at an $L$-element ULA, which has an interelement spacing of $d$.

For a $\mathrm{CW}$ waveform with frequency $f_{i}$, where $i$ is either 1 or 2 , the return signal from the $Q$ far-field targets, located at DOAs $\theta_{q}, q=1,2, \cdots, Q$, is expressed as

$$
\tilde{\mathbf{x}}_{i}(t)=\exp \left(j 2 \pi f_{i} t\right) \sum_{q=1}^{Q} \rho_{i}^{(q)}(t) \mathbf{a}_{i}\left(\theta_{q}\right)+\tilde{\mathbf{n}}_{i}(t),
$$

where $\rho_{i}^{(q)}(t)$ is the complex signal envelop which does not vary with the receive antennas but is in general frequency-dependent due to the different propagation phase delays. We assume $\rho_{i}^{(q)}(t)$ to be uncorrelated for different sources/targets due to independent source waveforms or, in the active sensing scenario, target motion or radar cross section (RCS) fluctuations. In addition, $\mathbf{a}_{i}\left(\theta_{q}\right)$ is the steering vector corresponding to $\theta_{q}$, expressed as

$$
\mathbf{a}_{i}\left(\theta_{q}\right)=\left[1, e^{-j \frac{2 \pi d}{\lambda_{i}} \sin \left(\theta_{q}\right)}, \ldots, e^{-j \frac{2 \pi(L-1) d}{\lambda_{i}} \sin \left(\theta_{q}\right)}\right]^{T}
$$

where $\lambda_{i}=c / f_{i}$ denotes the wavelength corresponding to $f_{i}$, and $c$ is the velocity of wave propagation. Furthermore, $\tilde{\mathbf{n}}_{i}(t)$ is the additive noise vector, whose elements are assumed to be spatially and temporally white.

After converting the received signal vector to baseband using the respective transmitted $\mathrm{CW}$ waveforms, followed by low-pass filtering, we obtain

$$
\mathbf{x}_{i}(t)=\sum_{q=1}^{Q} \rho_{i}^{(q)}(t) \mathbf{a}_{i}\left(\theta_{q}\right)+\mathbf{n}_{i}(t)=\mathbf{A}_{i} \mathbf{d}_{i}(t)+\mathbf{n}_{i}(t),
$$

where $\mathbf{A}_{i}=\left[\mathbf{a}_{i}\left(\theta_{1}\right), \cdots, \mathbf{a}_{i}\left(\theta_{Q}\right)\right]$ and $\mathbf{d}_{i}(t)=\left[\rho_{i}^{(1)}(t), \cdots, \rho_{i}^{(Q)}(t)\right]^{T}$. We denote the noise variance at the filter output as $\sigma_{n}^{(i)}$. 


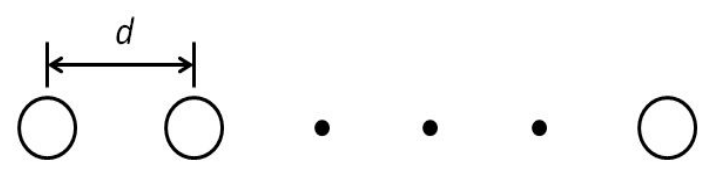

(a) A sparse ULA
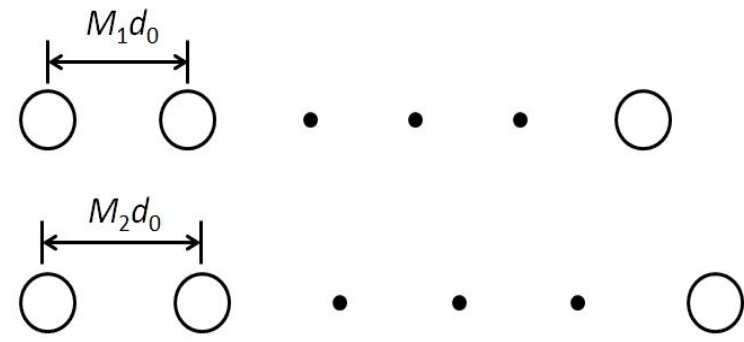

(b) Equivalent structure with two coprime frequencies

Figure 1. A sparse ULA with two coprime frequencies configuration.

In, ${ }^{11}$ a sufficient condition for alias-free DOA estimation is achieved by choosing the two frequencies to be coprime, i.e., the ratio equals the ratio between two coprime numbers. In this paper, the problem is considered in the context of coprime arrays. ${ }^{9}$ We assume that $d$ is an integer multiple of the half-wavelength at both frequencies, i.e., $M_{i}=2 d / \lambda_{i}$ are integers for $i=1,2$. As such, the ULA is sparse (spatially undersampled) at each frequency. $M_{1}$ and $M_{2}$ are chosen to be coprime, i.e., their greatest common divisor is one. In this case, we can rewrite the steering vectors in a frequency-independent form for better clarity,

$$
\mathbf{a}_{i}\left(\theta_{q}\right)=\left[1, e^{-j M_{i} \sin \left(\theta_{q}\right)}, \ldots, e^{-j M_{i}(L-1) d \sin \left(\theta_{q}\right)}\right]^{T} .
$$

It is clear that the DOA estimation problem is similar to the coprime arrays considered in. ${ }^{6}$ It is noted, however, that both subarrays share the same number of sensors, $L$.

\section{CO-ARRAY GENERATED FROM THE EQUIVALENT ARRAYS}

Without loss of generality, we assume $M_{1}<M_{2}$, illustrated in Fig. 1. The two equivalent subarrays are located at

$$
P=\left\{M_{1} l_{1} d_{0}, 0 \leq l_{1} \leq L-1\right\} \cup\left\{M_{2} l_{2} d_{0}, 0 \leq l_{2} \leq L-1\right\} .
$$

where $d_{0}$ denotes half-wavelength in a normalized frequency sense (i.e., no specific frequency is referred to). As such, the aperture of this equivalent coprime array structure is $M_{2}(L-1)$. In addition, the two uniform linear subarrays in the underlying problem have the same $L$ sensors, which align in the zeroth position and whenever $l / M_{2}$ is an integer. Therefore, there are $2 L-1-\left\lfloor(L-1) / M_{2}\right\rfloor$ equivalent physical sensors.

Because the two subarrays share the first sensor at the zeroth position, the self-lags of each subarray can be taken as cross-lags between every sensor of one subarray and the first sensor of the other subarray. That is, the self-lags form a subset of the cross-lags and, therefore, we only need to examine the cross-lags in determining the number of DOFs of the equivalent coprime array. The difference co-array between the two equivalent subarrays consists of the following cross-lags

$$
x\left(l_{1}, l_{2}\right)=\left\{M_{1} l_{1}-M_{2} l_{2}, 0 \leq l_{1} \leq L-1,0 \leq l_{2} \leq L-1\right\},
$$

and corresponding negative set is

$$
-x\left(l_{1}, l_{2}\right)=\left\{M_{2} l_{2}-M_{1} l_{1}, 0 \leq l_{1} \leq L-1,0 \leq l_{2} \leq L-1\right\} .
$$

It is apparent that $2 L^{2}-1$ lags are in set $\left\{-x\left(l_{1}, l_{2}\right) \cup x\left(l_{1}, l_{2}\right)\right\}$, which determines the global upper bound of the number of DOFs achievable from the difference co-array. The actual number of lags, $\eta$, is the number of 
unique lags in set $\left\{-x\left(l_{1}, l_{2}\right) \cup x\left(l_{1}, l_{2}\right)\right\}$, which may be smaller than $2 L^{2}-1$ if some lags are redundant. The number of DOFs achieved by a co-array of $\eta$ unique lags is equivalent to that of an array with $(\eta+1) / 2$ sensors.

When $M_{2} \geq L$, the number of unique lags of the co-array is given by

$$
\eta=2 L^{2}-1-\max \left\{0,2 L-1-M_{2}\right\} \min \left\{M_{1}+1,2 L-1-M_{1}\right\},
$$

where $M_{1}$ and $M_{2}$ are coprime, and $M_{1}<M_{2}$. The case of $M_{2}<L$ yields redundant lags in $x\left(l_{1}, l_{2}\right)$ and thus results in a smaller number of unique lags. For this reason, this case is not considered here.

It can be seen that, while $x\left(l_{1}, l_{2}\right)$ has $L^{2}$ distinct lags in line with the coprimality of $M_{1}$ and $M_{2}$, the actual value of $\eta$ depends on the values of $M_{1}$ and $M_{2}$. The reduction of the unique lags is due to the overlaps between $x\left(l_{1}, l_{2}\right)$ and $-x\left(l_{1}, l_{2}\right)$. In the following, we consider $(8)$ in three different cases:

- Case a: $\eta=2 L^{2}-1$ when $M_{2} \geq 2 L-1$. In this case, $\eta$ achieves the maximum value of $2 L^{2}-1$ irrespective of $M_{1}$. It should be noted, however, that a large value of $M_{2}$ may yield a high level sidelobe in the corresponding point spread function (PSF) of the co-array.

- Case b: $\eta=2 L^{2}-1-\left(2 L-1-M_{2}\right)\left(2 L-1-M_{1}\right)$ when $L \leq M_{1}<M_{2}$ and $L \leq M_{2}<2 L-1$.

- Case c: $\eta=2 L^{2}-1-\left(2 L-1-M_{2}\right)\left(M_{1}+1\right)$ when $1 \leq M_{1}<L$ and $L \leq M_{2}<2 L-1$.

For cases b and c, $\eta$ depends on both $M_{1}$ and $M_{2}$ and is maximized when $M_{1}=1$ (Case c) or $M_{1}=M_{2}-1$ (Case b). The latter is preferred because it yields a smaller frequency separation between $f_{1}$ and $f_{2}$.

For illustrative purpose, two co-array examples of different pairs of $M_{1}$ and $M_{2}$ are shown in Fig. 2, where the physical ULA has 6 sensors in both cases. It is clear that the difference co-arrays for all cases have more sensors than the sparse ULA. Nonetheless, the number of unique co-array lags is 41 as shown in Fig. 2(b) for the case of $M_{1}=5$ and $M_{2}=6$, whereas it increases to 71 in Fig. 2(a) when $M_{1}=10$ and $M_{2}=11$. Notice that overlapping lags are observed between $x\left(l_{1}, l_{2}\right)$ and $-x\left(l_{1}, l_{2}\right)$ in Fig. 2(b), and no lags overlap in Fig. 2(a).

\section{DOA ESTIMATION}

As we discussed earlier, a full number of unique lags is achieved in the resulting co-array by using the crosslags between the two subarrays. As such, compressive sensing (CS)-based DOA estimation can be performed based only on the cross-lag correlations without losing the available co-array DOFs. On the other hand, while exploiting both the self- and cross-lag correlations does not increase the number of DOFs of the co-array, the utilization of both self- and cross-lags makes better use of the observed data and thus may yield improved DOA estimation performance. In this case, however, the CS methods have to deal with the group sparsity for the selfand cross-lag correlations. In the following, we first discuss the DOA estimation from cross-lag observations, and the approach with both self- and cross-lags then follows.

The cross-lag correlation matrix between $\mathbf{x}_{1}(t)$ and $\mathbf{x}_{2}(t)$ is obtained as

$$
\mathbf{R}_{\mathbf{x x}}^{(1,2)}=\mathrm{E}\left[\mathbf{x}_{1}(t) \mathbf{x}_{2}^{H}(t)\right]=\mathbf{A}_{1} \mathbf{R}_{\mathbf{s s}}^{(1,2)} \mathbf{A}_{2}^{H}=\sum_{q=1}^{Q} \sigma_{q}^{(1,2)} \mathbf{a}_{1}\left(\theta_{q}\right) \mathbf{a}_{2}^{H}\left(\theta_{q}\right),
$$

where $\mathbf{R}_{\mathbf{s}}^{(1,2)}=\mathrm{E}\left[\mathbf{d}_{1}(t) \mathbf{d}_{2}^{H}(t)\right]=\operatorname{diag}\left(\left[\sigma_{1}^{(1,2)}, \ldots, \sigma_{Q}^{(1,2)}\right]\right)$ is the cross-correlation matrix between the source signals at the two frequencies. Note that $\sigma_{q}^{(1,2)}, p=1, \ldots, Q$, in general, takes a complex value. In addition, because $\mathbf{R}_{\mathbf{x x}}^{(2,1)}=\left(\mathbf{R}_{\mathbf{x x}}^{(1,2)}\right)^{H}$, the term $\mathbf{R}_{\mathbf{x x}}^{(2,1)}$ does not carry additional information and thus is not considered in the CS-based DOA estimation.

Vectorizing $\mathbf{R}_{\mathbf{x x}}^{(1,2)}$ yields an $L^{2} \times 1$ vector

$$
\mathbf{z}^{(1,2)}=\operatorname{vec}\left(\mathbf{R}_{\mathbf{x x}}^{(1,2)}\right)=\tilde{\mathbf{A}}^{(1,2)} \mathbf{b}^{(1,2)},
$$


- $\quad$ Lags in $x\left(l_{1}, l_{2}\right) \quad \mathrm{x} \quad$ Holes

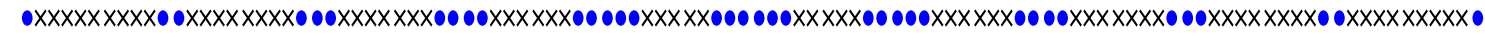
$\begin{array}{llllllllllllllllllllll}-50 & -45 & -40 & -35 & -30 & -25 & -20 & -15 & -10 & -5 & 0 & 5 & 10 & 15 & 20 & 25 & 30 & 35 & 40 & 45 & 50 & 55\end{array}$

- Lagsin co-array $\quad \times \quad$ Holes

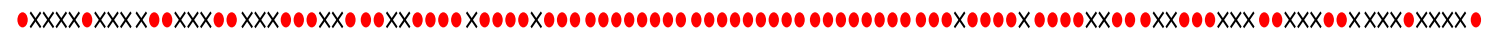
$\begin{array}{lllllllllllllllllllllll}-55 & -50 & -45 & -40 & -35 & -30 & -25 & -20 & -15 & -10 & -5 & 0 & 5 & 10 & 15 & 20 & 25 & 30 & 35 & 40 & 45 & 50 & 55\end{array}$

(a) $M_{1}=10$ and $M_{2}=11$

- Lags in $x\left(l_{1}, l_{2}\right) \quad \mathrm{x}$ Holes $\quad 0$ overlaps between $x\left(l_{1}, l_{2}\right)$ and $-x\left(l_{1}, l_{2}\right)$

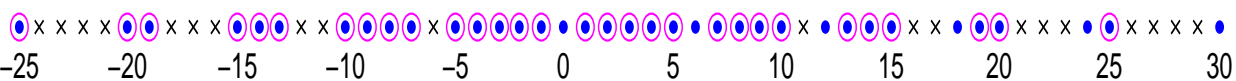

- Lagsin co-array $\times$ Holes

$0 \times x \times x 00 \times x \times 000 \times x 0000 \times 000000000000000000000 \times 0000 \times x 000 \times x \times 00 \times x \times x=$

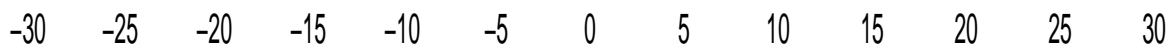

(b) $M_{1}=5$ and $M_{2}=6$

Figure 2. Co-array examples for different choices of $M_{1}$ and $M_{2}(L=6)$.

where $\tilde{\mathbf{A}}^{(i, l)}=\left[\tilde{\mathbf{a}}_{(i, l)}\left(\theta_{1}\right), \ldots, \tilde{\mathbf{a}}_{(i, l)}\left(\theta_{Q}\right)\right], \tilde{\mathbf{a}}_{(i, l)}\left(\theta_{q}\right)=\mathbf{a}_{i}^{*}\left(\theta_{q}\right) \bigotimes \mathbf{a}_{l}\left(\theta_{q}\right), \mathbf{b}^{(i, l)}=\left[\sigma_{1}^{(i, l)}, \ldots, \sigma_{Q}^{(i, l)}\right]^{T}$. The DOA estimates can formulated as the following constrained $l_{1}$-norm minimization problem,

$$
\begin{aligned}
& \hat{\mathbf{b}}^{(1,2)}=\arg \min _{\mathbf{b}^{(1,2)}}\left\|\mathbf{b}^{(1,2)}\right\|_{1} \\
& \text { s.t. }\left\|\mathbf{z}^{(1,2)}-\tilde{\mathbf{A}}^{(1,2)} \mathbf{b}^{(1,2)}\right\|_{2}<\epsilon_{1},
\end{aligned}
$$

where $\epsilon_{1}$ is a user-specific bound. This type of problems has been the objective of intensive studies in the area of CS, and a number of effective numerical computation methods has been developed to obtain the solution by solving a corresponding overdetermined problem defined in a dense search grid. In this paper, we use the batch Lasso method, but other methods may also be used.

When considering both self- and cross-lags for the DOA estimation, we further obtain

$$
\mathbf{R}_{\mathbf{x x}}^{(i)}=\mathrm{E}\left[\mathbf{x}_{i}(t) \mathbf{x}_{i}^{H}(t)\right]=\mathbf{A}_{i} \mathbf{R}_{\mathbf{s s}}^{(i)} \mathbf{A}_{i}^{H}+\sigma_{n}^{(i)} \mathbf{I}_{L}=\sum_{q=1}^{Q} \sigma_{q}^{(i)} \mathbf{a}_{i}\left(\theta_{q}\right) \mathbf{a}_{i}^{H}\left(\theta_{q}\right)+\sigma_{n}^{(i)} \mathbf{I}_{L}
$$

where $i$ takes a value of either 1 or 2 . In the above expressions, $\mathbf{R}_{\mathbf{s s}}^{(i)}=\mathrm{E}\left[\mathbf{d}_{i}(t) \mathbf{d}_{i}^{H}(t)\right]=\operatorname{diag}\left(\left[\sigma_{1}^{(i)}, \ldots, \sigma_{Q}^{(i)}\right]\right)$ is the source covariance matrix where $\sigma_{q}^{(i)}$ is real and positive. Vectorizing $\mathbf{R}_{\mathbf{x x}}^{(i)}$ yields an $L^{2} \times 1$ vector,

$$
\mathbf{z}^{(i)}=\operatorname{vec}\left(\mathbf{R}_{\mathbf{x x}}^{(i)}\right)=\tilde{\mathbf{A}}^{(i)} \mathbf{b}^{(i)}+\sigma_{n}^{(i)} \mathbf{i}
$$

where $\tilde{\mathbf{A}}^{(i)}=\left[\tilde{\mathbf{a}}_{(i)}\left(\theta_{1}\right), \ldots, \tilde{\mathbf{a}}_{(i)}\left(\theta_{Q}\right)\right], \tilde{\mathbf{a}}_{(i)}\left(\theta_{q}\right)=\mathbf{a}_{i}^{*}\left(\theta_{q}\right) \bigotimes \mathbf{a}_{i}\left(\theta_{q}\right), \mathbf{b}^{(i)}=\left[\sigma_{1}^{(i)}, \ldots, \sigma_{Q}^{(i)}\right]^{T}$.

As we can observe from the above discussion, elements of $\mathbf{b}^{(i)}$ and $\mathbf{b}^{(i, l)}$, respectively depicted in (13) and (10), share the same sparsity support corresponding to the directions of the $Q$ targets but generally take different values. As such, the DOA estimation problem must be solved in the group sparse context. ${ }^{9}$ In this paper, we use the group Lasso to locate the non-zero entries of $\mathbf{b}^{(i)}$ and $\mathbf{b}^{(i, l)}$ for DOA estimation. 
Stacking vectors $\mathbf{z}^{(1)}, \mathbf{z}^{(2)}$, and $\mathbf{z}^{(1,2)}$ as $\mathbf{z}=\left[\left(\mathbf{z}^{(1)}\right)^{T},\left(\mathbf{z}^{(2)}\right)^{T},\left(\mathbf{z}^{(1,2)}\right)^{T}\right]^{T}$. Then, we can combine (10) and (13) into a single expression as,

$$
\mathbf{z}=\tilde{\mathbf{A}} \mathbf{b}+\tilde{\mathbf{I}} \boldsymbol{\sigma}_{n},
$$

where $\mathbf{b}=\left[\left(\mathbf{b}^{(1)}\right)^{T},\left(\mathbf{b}^{(2)}\right)^{T},\left(\mathbf{b}^{(1,2)}\right)^{T}\right]^{T}, \tilde{\mathbf{A}}$ is a block diagonal matrix consisting of $\tilde{\mathbf{A}}^{(1)}, \tilde{\mathbf{A}}^{(2)}$ and $\tilde{\mathbf{A}}^{(1,2)}$. In addition, $\boldsymbol{\sigma}_{n}=\left[\sigma_{n}^{(1)}, \sigma_{n}^{(2)}\right]^{T}$, and $\tilde{\mathbf{I}}$ is a $3 L^{2} \times 2$ matrix whose first $\left[\mathbf{i}^{T}, \mathbf{0}^{T}, \mathbf{0}^{T}\right]^{T}$, where $\mathbf{0}$ denotes the all zero vector of dimension $L^{2} \times 1$, and the second column is $\left[\mathbf{0}^{T}, \mathbf{i}^{T}, \mathbf{0}^{T}\right]^{T}$. The estimation of $\mathbf{b}$ can be formulated as the following minimization problem,

$$
\begin{aligned}
& \quad \hat{\mathbf{b}}=\arg \min _{\mathbf{b}}\|\xi(\mathbf{b})\|_{1} \\
& \text { s.t. } \quad\left\|\mathbf{z}-\tilde{\mathbf{A}} \mathbf{b}-\sigma_{n} \tilde{\mathbf{I}}\right\|_{2}<\epsilon_{2},
\end{aligned}
$$

where $\epsilon_{2}$ is a user-specific bound, and $\xi($.$) is an operation that obtains the l_{2}$ norm of the three-element entries corresponding to each spatial position. We use the group Lasso to solve a corresponding problem defined in a dense search grid.

\section{SIMULATION RESULTS}

For illustration, we consider a ULA of $L=6$ antenna sensors. $Q=19$ targets, which are uniformly distributed between $-60^{\circ}$ and $60^{\circ}$, are assumed. The noise power at the two frequencies are assumed to be the same, and the input SNR is $-10 \mathrm{~dB}$ for all sources are assumed to be identical. The number of snapshots is 2000, and the grid size for space angle search is $0.1^{\circ}$. The phase difference between the received signal corresponding to the two frequencies is independently and uniformly distributed over $[0,2 \pi]$.

We first show the importance of using coprime frequencies to maximize the DOFs. To demonstrate this fact, we show in Fig. 3 the results for different pairs of $M_{1}$ and $M_{2}$. Specifically, $M_{1}=4$ and $M_{2}=6$ are used in Fig. 3(a). Only the cross-lags are used. In this case, $M_{1}$ and $M_{2}$ are not coprime and the number of resulting unique lags is $\eta=29$. As such, the yielding co-array does not resolve the 19 sources. As a comparison, in the case of $M_{1}=5$ and $M_{2}=6$, which is depicted in Fig. 3(b), there are $\eta=41$ unique lags, and all the 19 sources are resolved.

In the next example, we consider the advantage of using both self- and cross-lags as compared to the case when only the cross-lag is used. Fig. 4(a) shows improved DOA estimation performance as the result of using both self- and cross-lags as compared to the cross-lag only results replicated in Fig. 4(b).

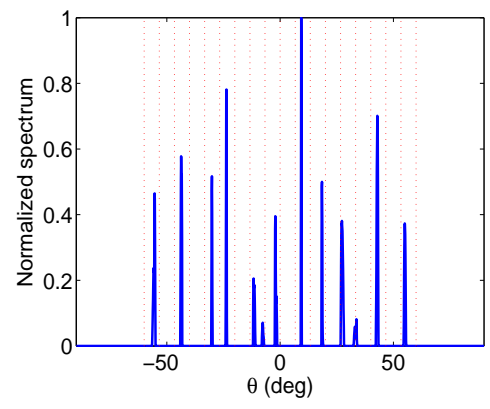

(a) $M_{1}=4, M_{2}=6$

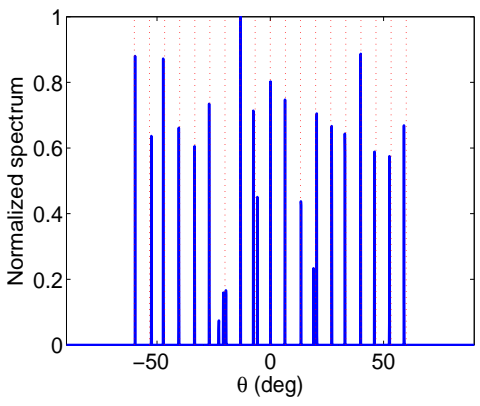

(b) $M_{1}=5, M_{2}=6$

Figure 3. Spatial spectra estimated for different choice of $M_{1}$ and $M_{2}$.

\section{CONCLUSION}

In this paper, we examined the coprime array construction using a sparse uniform linear array with two coprime frequencies. We derived the analytical expressions of the number of unique lags of the yielding co-array to determine the number of detectable sources. It was shown that full degrees-of-freedom are achieved by using only the cross-lag correlations, but the combined use of the self- and cross-lags improves the DOA estimation performance. 


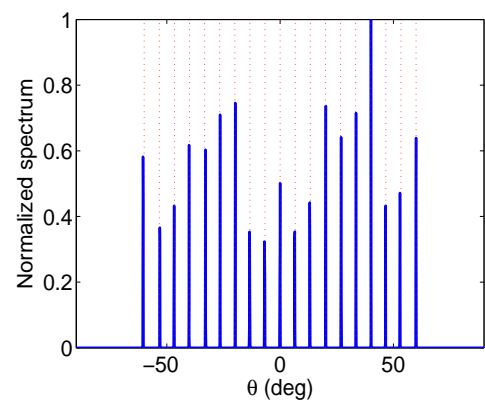

(a) Using self- and cross-lags

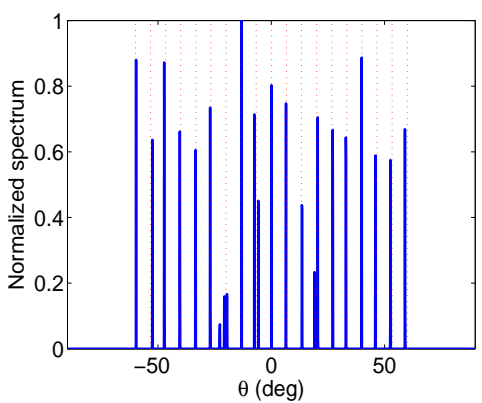

(b) Using cross-lags only

Figure 4. Spatial spectra estimated for $M_{1}=5, M_{2}=6$.

\section{ACKNOWLEDGMENTS}

This work was supported in part by the Office of Naval Research (ONR) under Grant N00014-13-1-0061.

\section{REFERENCES}

1. R. O. Schmidt, "Multiple emitter location and signal parameter estimation," IEEE Trans. Antennas Propagat., vol. 34, no. 3, pp. 276-280, March 1986.

2. R. Roy and T. Kailath, "ESPRIT - Estimation of signal parameters via rotation invariance techniques," IEEE Trans. Acoust., Speech, Signal Proc., vol. 17, no. 7, pp. 984-995, July 1989.

3. R. T. Hoctor and S. A. Kassam, "The unifying role of the co-array in aperture synthesis for coherent and incoherent imaging," Proc. IEEE, vol. 78, no. 4, pp. 735-752, April 1990.

4. A. Moffet, "Minimum-redundancy linear arrays," IEEE Trans. Antennas Propagat., vol. 16, no. 2, pp. 172-175, March 1968.

5. P. Pal and P. P. Vaidyanathan, "Nested Arrays: A novel approach to array processing with enhanced degrees of freedom," IEEE Trans. Signal Proc., vol. 58, no. 8, pp. 4167-4181, Aug. 2010.

6. P. P. Vaidyanathan and P. Pal, "Sparse sensing with co-prime samplers and arrays," IEEE Trans. Signal Proc., vol. 59, no. 2, pp. 573-586, Feb. 2011.

7. P. Pal and P. P. Vaidyanathan, "Coprime sampling and the MUSIC algorithm," in Proc. IEEE Digital Signal Proc. Workshop and IEEE Signal Proc. Education Workshop, Sedona, AZ, Jan. 2011.

8. S. Qin, Y. D. Zhang, and M. G. Amin, "Generalized coprime array configurations," in Proc. IEEE Sensor Array and Multichannel Signal Processing Workshop, A Coruña, Spain, June 2014.

9. Y. D. Zhang, M. G. Amin, F. Ahmad, and B. Himed, "DOA estimation using a sparse uniform linear array with two CW signals of co-prime frequencies," in Proc. IEEE Int. Workshop on Comp. Adv. in Multi-Sensor Adaptive Proc., Saint Martin, Dec. 2013.

10. Y. D. Zhang, S. Qin, and M. G. Amin, "DOA estimation exploiting coprime arrays with sparse sensor spacing," in Proc. IEEE ICASSP, Florence, Italy, May 2014.

11. M. G. Amin, "Sufficient conditions for alias-free direction of arrival estimation in periodic spatial spectra, IEEE Trans. Antennas Propagat., vol. 41, no. 4, pp. 508-511, April 1993.

12. P. Pal and P. P. Vaidyanathan, "On application of LASSO for sparse support recovery with imperfect correlation awareness," in Proc. Asilomar Conf. Signals, Systems and Computers, Pacific Grove, CA, Nov. 2012.

13. Y. D. Zhang, M. G. Amin, and B. Himed, "Sparsity-based DOA estimation using co-prime arrays," in Proc. IEEE ICASSP, Vancouver, Canada, May 2013. 